Biotempo (Lima)

ORIGINAL ARTICLE / ARTÍCULO ORIGINAL

SPATIO-TEMPORAL VARIATION OF THE MARINE PHYTOPLANCTON ON THE ALBACORA PLATFORM, LOT Z1, TUMBES, PERU

\title{
VARIACIÓN ESPACIO - TEMPORAL DEL FITOPLANCTON MARINO EN LA PLATAFORMA ALBACORA, LOTE Z1, TUMBES, PERÚ
}

\author{
Sandra Falero-Alama ${ }^{1}$ \& Flor de María Madrid-Ibarra ${ }^{1}$
}

1 Instituto de Recursos Naturales y Ecología (IRNE) Universidad Ricardo Palma, Lima-Perú.

sandraclaudettef@gmail.com; flordemaria.madrid@urp.edu.pe

\begin{abstract}
The present investigation was carried out on the Albacora platform of Lot Z1, 10 nautical miles from the coast of the districts of Corrales and La Cruz, Province of Tumbes - Tumbes, Peru and consisted in evaluating the quarterly spatio-temporal variation of the marine phytoplankton of the year 2014. The environmental variables were taken as sea surface temperature, salinity, $\mathrm{pH}$ and dissolved oxygen. 100 samples were collected in total. The phytoplankton samples were collected on the surface, by means of a network of $75 \mathrm{u}$ of opening with a diameter of $20 \mathrm{~cm}$, by surface trawling in a craft fishing vessel at a speed of 3 knots for $5 \mathrm{~min}$. A total of 201 species was recorded between diatoms and dinoflagellates, with diatoms predominating with 119 species. The spatio-temporal variation of the phytoplankton registered greater richness and abundance in stations E-4 $(2 \mathrm{~km}$, southwest of the Platform) and E-5 (2 km, southeast of the Platform) during the second quarter at an average surface temperature of $25.5^{\circ} \mathrm{C}$, salinity $33.2 \mathrm{UPS}, \mathrm{pH} 8.1$ and Dissolved Oxygen $5.9 \mathrm{mg} \cdot \mathrm{L}^{-1}$, values that are within the water quality standards (ECA), according to $\mathrm{DS}^{\circ} 002-2008$ MINAM (Ministry of the Environment) of Peru. Regarding phytoplankton diversity indices, the dominance of diatoms over dinoflagellates in E-1 stations (500 m northeast of the Platform, in favor of the current), E-2 (500 m northwest) of the Platform, against the current) and E-3 (Plataforma Albacora), was recognized during all the quarters of the year. The presence of Chaetoceros coarctatus Lauder 1864, Guinardia striata (Stolterfoth) Hasle in Hasle \& Syvertsen 1996 and Ceratium breve (Ostenfeld \& Schmidt) Schröder 1906 related to the increase in temperature and typical of Surface Equatorial Waters (AES) are reported as phytoplankton bioindicators.
\end{abstract}

Key words: Diversity indices - Phytoplankton - Spatial-temporal variation

\section{RESUMEN}

La presente investigación se realizó en la plataforma Albacora del Lote Z1, a 10 millas náuticas de la costa de los distritos de Corrales y La Cruz, Provincia de Tumbes - Tumbes, Perú y consistió en evaluar la variación espaciotemporal trimestral del fitoplancton marino del ańo 2014; se tomaron las variables ambientales como temperatura superficial del mar, salinidad, $\mathrm{pH}$ y oxígeno disuelto. Se obtuvieron 100 muestras en total. Las muestras de fitoplancton 
se recolectaron en superficie, mediante una red de $75 \mathrm{u}$ de abertura con un diámetro de $20 \mathrm{~cm}$, por arrastre superficial en una embarcación de pesca artesanal a una velocidad de 3 nudos por 5 min. Se registró un total de 201 especies entre diatomeas y dinoflagelados, predominando las diatomeas con 119 especies; obteniéndose como resultados que la variación espacio-temporal del fitoplancton registró mayor riqueza y abundancia en las estaciones E-4 (2 Km, al suroeste de la Plataforma) y E-5 (2 Km, al sureste de la Plataforma) durante el segundo trimestre, a una temperatura superficial promedio de $25,5^{\circ} \mathrm{C}$, salinidad 33,2 UPS, $\mathrm{pH} 8,1$ y Oxígeno Disuelto 5,9 $\mathrm{mg} \cdot \mathrm{L}^{-1}$, valores que se encuentran dentro de los estándares de calidad de agua (ECA), según el DS 002-2008-MINAM (Ministerio del Ambiente) del Perú. En cuanto a los índices de diversidad del fitoplancton reconocen el dominio de las diatomeas sobre los dinoflagelados en las estaciones E-1 (a $500 \mathrm{~m}$ al noreste de la Plataforma, a favor de la corriente), E-2 (a $500 \mathrm{~m}$ al noroeste de la Plataforma, en contra de la corriente) y E-3 (Plataforma Albacora), durante todos los trimestres del año. Se reportan como bioindicadores del fitoplancton a Chaetoceros coarctatus Lauder 1864, Guinardia striata (Stolterfoth) Hasle in Hasle \& Syvertsen 1996 y Ceratium breve (Ostenfeld \& Schmidt) Schröder 1906 relacionados con el incremento de la temperatura y típicas de Aguas Ecuatoriales Superficiales (AES).

Palabras clave: Fitoplancton - Índices de diversidad - Variación espacio-temporal

\section{INTRODUCCIÓN}

El ecosistema marino del Noroeste peruano forma parte de la Corriente Ecuatorial y se caracteriza por ser de aguas cálidas que aparecen entre los meses de diciembre a abril. Esta corriente provoca el aumento de la temperatura superficial del mar y sus meses de mayor intensidad son de enero abril, posee un flujo estrecho costero de norte a sur que proviene de la cuenca de panamá, y baja hasta las costas de Ecuador y Perú (INOCAR, 2017). La diversidad planctónica que presenta el mar de Tumbes, cuya temperatura corresponde a aguas tropicales y ecuatoriales, muestra una gran diversidad de hábitats y nichos ecológicos; teniendo al fitoplancton como representante de la cadena trófica. En la provincia de Tumbes, se ha desarrollado la actividad de extracción de hidrocarburos mediante el uso de plataformas marinas, como la Plataforma Albacora que se encuentra en el Lote Z1 ubicada a 10 millas náuticas de la costa norte. Las operaciones de exploración, perforación y extracción de hidrocarburos alteran las condiciones físico-químicas y biológicas de los ecosistemas marinos. Estos parámetros son determinantes para la estabilidad de la biodiversidad (Ruppert et al., 1996; Morón, 2000; Coello et al., 2010; Montero, 2013).

Los cambios que se producen en el espacio y en el tiempo generan variaciones en la composición del fitoplancton, determinando que especímenes se repiten en un mismo espacio-tiempo de manera que se puedan considerar como potenciales bioindicadores (Lanza-Espino et al., 2011). El fitoplancton es utilizado como un bioindicador muy promisorio debido a sus ciclos vitales cortos en el medio marino; además reflejan su sensibilidad a los cambios de temperatura y salinidad, siendo buenos indicadores oceanográficos (Zuta \& Guillén, 1970; Mohan et al., 2006; Delgado, 2018). Este responde rápidamente a los cambios que pueden ocurrir en las masas de agua por procesos naturales o antrópicos.

Algunas especies son buenos indicadores biológicos de las masas de agua como Aguas Tropicales Superficiales (ATS) y Aguas Ecuatoriales Superficiales (AES). Es importante también relacionar la información existente ya publicada, sobre la comunidad planctónica, y reconocer las especies que se presentan estacional y temporalmente (Delgado et al., 2015). Cumpliendo con las obligaciones que tienen las empresas de actividades extractivas en la provincia de Tumbes con el estado peruano, se llevan a cabo planes de control y seguimiento de la calidad ambiental y calidad del agua marina en las áreas que se realizan operaciones de exploración, perforación y extracción de hidrocarburos. El monitoreo y la evaluación de la calidad del agua, está basado en las propiedades físico-químicas y biológicas del agua, cuya meta es brindar información útil para la gestión ambiental (INVEMAR, 2003).

La presente investigación evaluó la variación espacio - temporal del fitoplancton marino en la plataforma Albacora, Lote Z1, Tumbes, Perú.

\section{MATERIALES Y MÉTODOS}

El área de estudio se ubica a 10 millas náuticas (mn) de la línea de costa entre los distritos de Corrales y La Cruz (Provincia de Tumbes). Se tomaron datos de cinco estaciones hasta un radio de $2 \mathrm{Km}$ de la plataforma (Fig. 1). Las estaciones fueron georreferenciadas con el uso del GPS y escogidas según lo indicado en Tabla 1. 


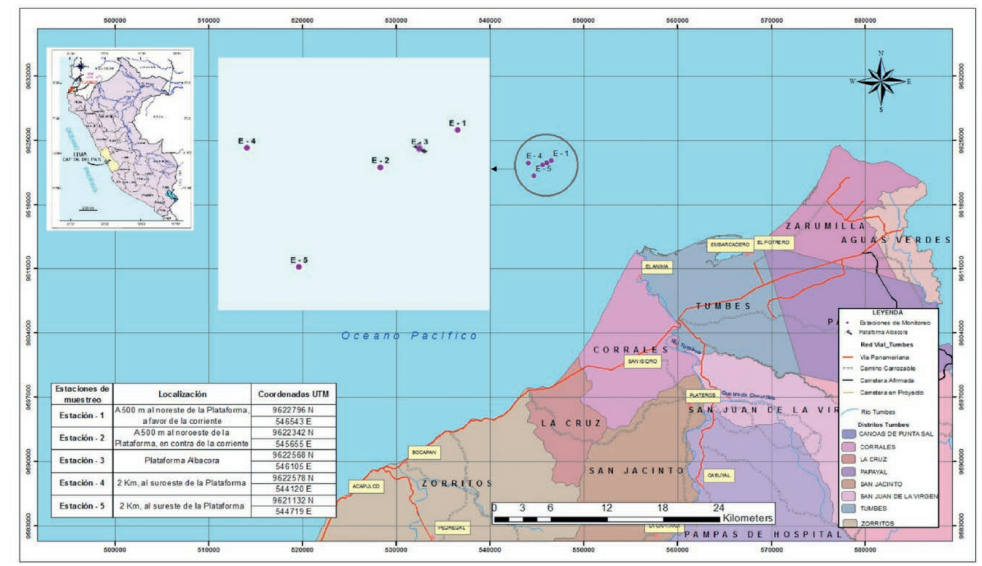

Figura 1. Ubicación de las estaciones de muestreo en la plataforma Albacora, Lote Z1, Tumbes, Perú.

Tabla 1. Estaciones de muestreo y sus coordenadas UTM.

\begin{tabular}{lll}
\hline Estaciones de muestreo & Localización & Coordenadas UTM \\
\hline Estación -1 (E-1) & A $500 \mathrm{~m}$ al noreste de la Plataforma, a favor de la corriente & $9622796 \mathrm{~N}, 546543 \mathrm{E}$ \\
Estación - 2 (E-2) & A $500 \mathrm{~m}$ al noroeste de la Plataforma, en contra de la corriente & $9622342 \mathrm{~N}, 545655 \mathrm{E}$ \\
Estación - 3 (E-3) & Plataforma Albacora & $9622568 \mathrm{~N}, 546105 \mathrm{E}$ \\
Estación -4 (E-4) & 2 Km, al suroeste de la Plataforma & $9622578 \mathrm{~N}, 544120 \mathrm{E}$ \\
Estación - 5 (E-5) & 2 Km, al sureste de la Plataforma & $9621132 \mathrm{~N}, 544719 \mathrm{E}$ \\
\hline
\end{tabular}

\section{Toma de muestra en Campo}

Se hizo desde una embarcación de pesca artesanal de la localidad de Zorritos. Se recolectaron 100 muestras en total y la metodología utilizada fue la de Rice \& APHA (2012) con algunas modificaciones en campo (SMEWW $10200-$ $\mathrm{C} 1, \mathrm{~F} 2)$. Adicionalmente se tomó la temperatura, salinidad, $\mathrm{pH}$ y oxígeno disuelto "in situ" con un multiparámetro de marca Hanna ${ }^{(\mathrm{R})}$ (Figura 2) en cada estación de muestreo.

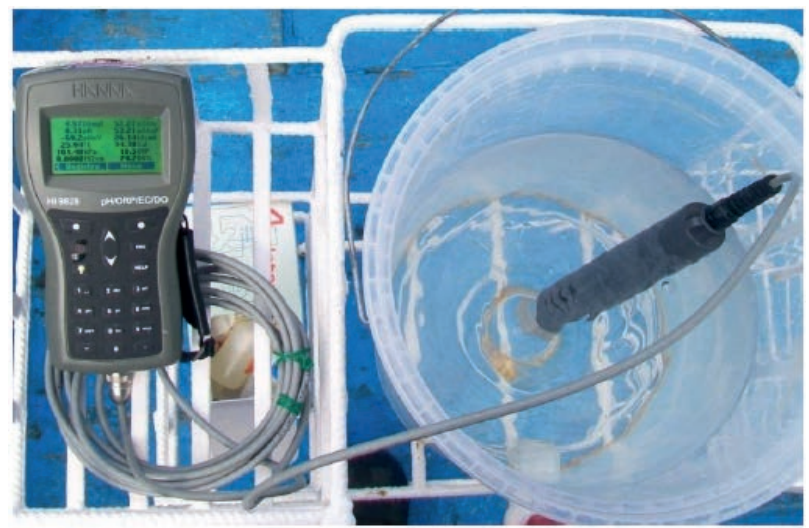

Figura 2. Medición de las variables ambientales con el uso del Multiparámetro de marca Hanna.

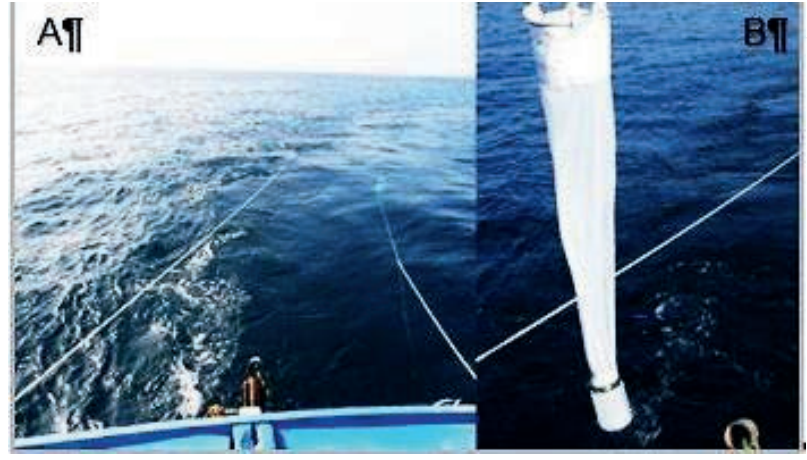

Figura 3. Colecta del plancton: El lado izquierdo (A) muestra las redes del plancton arrastradas desde la embarcación. El lado derecho (B) muestra la red de fitoplancton con el material recolectado.

\section{Procedimiento y análisis de datos}

Las muestras del fitoplancton se analizaron mediante la observación al microscopio empleando la técnica de recuento de Utermöhl (1958). Los resultados se expresan en número de células/Litro (abundancia). La identificación taxonómica de las especies se realizó con el uso de claves taxonómicas especializadas en el tema (Balech \& Ferrando, 1964; Pesantes, 1983; Round et al., 2000). 
Se construyó una matriz de la riqueza y abundancia de especies por meses y por estaciones, además, se graficaron los valores por trimestre con el uso del programa Excel. Los índices de diversidad se obtuvieron con el uso del programa estadístico PAST (PAleontological STatistics Software versión 3.0), asimismo se trabajaron los índices de Shannon- Wiener, Simpson y Pielou según Magurran (1988) y Hernández \& Alcolado (2014).

\section{RESULTADOS Y DISCUSIÓN}

El desplazamiento de aguas superficiales hacia la zona costera fue evidente por la gran riqueza de especies observadas durante todo el año 2014, determinándose el dominio absoluto de las diatomeas sobre los dinoflagelados.

Durante el primer trimestre se reportó un total de 110 especies en el fitoplancton evaluado, la abundancia total fue de $600.070 \mathrm{cel} \cdot \mathrm{L}^{-1}$ entre diatomeas y dinoflagelados. Las diatomeas se registraron con el dominio del genero Chaetoceros Ehrenberg, 1844 con 19 especies, coincidiendo con Vera (2014) y Basilio (2014) que mencionan a Chaetoceros como dominante en aguas superficiales. La especie más abundante fue Chaetoceros curvisetus Cleve, 1889 con $217.640 \mathrm{cel} \cdot \mathrm{L}^{-1}$ (Tabla 2), similar a lo que mencionan Ordinola (2017), Ruiz (2017) y Tarazona et al. (2003), como especie típica de la zona norte del Perú. Las estaciones con mayor número de especies fueron E-1 y E-2, la estación E-3 presentó menor riqueza y abundancia, no obstante la recuperación de la riqueza y la abundancia se observa en las estaciones E-4 y E-5 (Figuras 4-5).

En cuanto a los dinoflagelados registraron al género Prorocentrum Ehrenberg, 1833 con 3 especies, con su especie más abundante Prorocentrum micans Ehrenberg, 1834; con 3.720 cel. $\mathrm{L}^{-1}$ (Tabla 3), coincidiendo con los autores Torres \& Tapia (2000), Tapia (2012) y Ruiz (2017), que mencionan a estos géneros como los más frecuentes y abundantes de los dinoflagelados. Asimismo Tarazona et al. (2003) mencionan que los dinoflagelados dominantes en la zona en su mayoría corresponden a los géneros Ceratium Schrank, 1793 y Protoperidinium Bergh, 1881, géneros reportados en nuestra investigación. La estación con mayor riqueza y abundancia fue E-4 (Figuras 4-5). Las variables ambientales se registraron de la siguiente manera: La temperatura superficial del mar osciló entre $26,2^{\circ} \mathrm{C}$ a $27,9^{\circ} \mathrm{C}$; la salinidad entre $30,4 \mathrm{UPS}$ y 33,9 UPS; el pH por debajo de 8 y el Oxígeno disuelto entre 5,4 a 6,4 $\mathrm{mg} \cdot \mathrm{L}^{-1}$, valores que se encuentran dentro de los Estándares de Calidad Ambiental (ECA) de agua marina (MINAM, 2008).

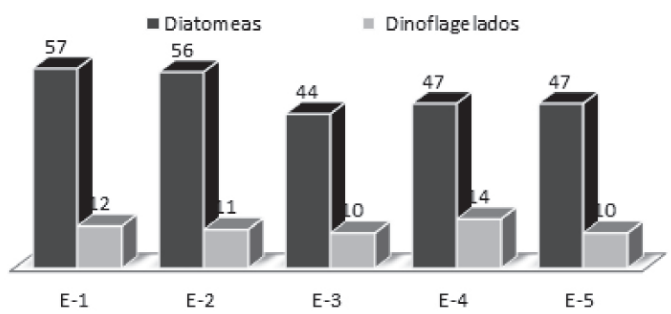

Figura 4. Riqueza del Fitoplancton registrado en el primer trimestre en las estaciones de muestreo. E-1= A $500 \mathrm{~m}$ al noreste de la Plataforma, a favor de la corriente; $\mathrm{E}-2=\mathrm{A} 500 \mathrm{~m}$ al noroeste de la Plataforma, en contra de la corriente; E-3= Plataforma Albacora; E-4= $2 \mathrm{Km}$, al suroeste de la Plataforma; E-5 $=2 \mathrm{Km}$, al sureste de la Plataforma.

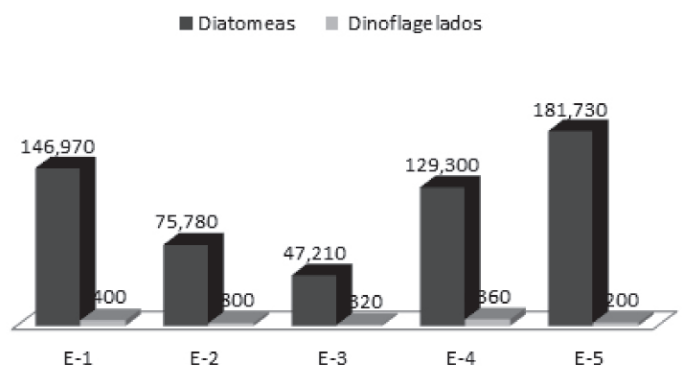

Figura 5. Abundancia (cel. $\left.\mathrm{L}^{-1}\right)$ del Fitoplancton registrado en el primer trimestre en las estaciones de muestreo. $\mathrm{E}-1=\mathrm{A} 500 \mathrm{~m}$ al noreste de la Plataforma, a favor de la corriente; E-2= A $500 \mathrm{~m}$ al noroeste de la Plataforma, en contra de la corriente; E-3= Plataforma Albacora; E-4= 2 $\mathrm{Km}$, al suroeste de la Plataforma; E-5=2 Km, al sureste de la Plataforma.

En el segundo trimestre (abril-junio 2014) el fitoplancton evaluado presentó un total de 101 especies entre diatomeas y dinoflagelados. La abundancia entre diatomeas y dinoflagelados registró un total de 2 844,530 cel. $\mathrm{L}^{-1}$. Las diatomeas que dominaron fueron del genero Chaetoceros con 26 especies, similar a los que menciona el autor Armijos (2007) con el dominio de este género en las evaluaciones superficiales de aguas cálidas, y la especie más abundante Chaetoceros lorenzianus Grunow, 1863 con $351.010 \mathrm{cel} \cdot \mathrm{L}^{-1}$ (Tabla 4). Las estaciones con mayor número de especies e individuos fueron E-4 y E-5, en cuanto la estación E-3 presentó mayor riqueza y menor abundancia (Figuras 6-7). En cuanto a los dinoflagelados registraron la especie más abundante Scrippsiella trochoidea (F.Stein) A.R.Loeblich III, 1976 con 20.400 cel.L ${ }^{-1}$ (Tabla 5), coincidiendo con Armijos (2007) debido a su presencia en el mes de abril. La estación con mayor riqueza y abundancia fue E-4 (Figuras 6-7). 
Tabla 2. Composición y abundancia (cel $\left.\cdot \mathrm{L}^{-1}\right)$ de Diatomeas registradas por estación en el primer trimestre enero marzo 2014. Se resaltan las especies con los valores más altos de abundancia.

\begin{tabular}{|c|c|c|c|c|c|c|c|}
\hline & DIATOMEAS & $E-1$ & E.2 & E-3 & $\mathrm{E} \cdot 4$ & E.5 & TOTAL \\
\hline 1 & Actinoorclus sp. & 0 & 40 & 0 & 40 & 200 & 280 \\
\hline 2 & Amphiprora sp. & $240^{\circ}$ & 0 & 0 & 200 & 0 & $440^{\circ}$ \\
\hline 3 & Amphora sp. & 0 & 40 & 0 & 0 & 0 & 40 \\
\hline 4 & Asterompholus orrochne & 0 & 0 & 40 & 0 & 0 & 40 \\
\hline 5 & Asterompholus sp. & & 0 & 0 & 40 & 0 & 40 \\
\hline 6 & Bacteristrstrum delichatulum & 1200 & 0 & 720 & 0 & 4160 & 6080 \\
\hline 7 & Bacterlistrtum hyolinum & 0 & 280 & 0 & $\circ$ & 0 & 280 \\
\hline 8 & Bacteriastrum sp. & o & 560 & 。 & $\circ$ & 0 & 560 \\
\hline 9 & Cerotoulina pelagica & 1160 & 320 & 160 & 280 & 280 & 2200 \\
\hline 10 & Cerotaulina bicornis & 120 & 0 & 240 & 340 & 640 & 1340 \\
\hline 11 & Chaetoceros affinis & 440 & 1240 & 。 & $\circ$ & 0 & 1680 \\
\hline 12 & Chaetoceros borealis & 0 & 160 & 0 & o & 0 & 160 \\
\hline 13 & Chaetoceros brevis & 1600 & 400 & 160 & $\circ$ & 80 & 2240 \\
\hline 14 & Choetoceros $f$ f. attanticus & 80 & 160 & 。 & 320 & 0 & 560 \\
\hline 15 & Chaetoceros compressus & 10840 & 2880 & 960 & 4000 & 1080 & 19760 \\
\hline 16 & chaetoceros constritictus & 0 & 200 & 0 & 0 & 0 & 200 \\
\hline 17 & Chaetoceros costatus & 1000 & o & 。 & 1240 & 0 & 2240 \\
\hline 18 & Cheetoceros curvisetus & 33080 & 14360 & 15160 & 41760 & 113280 & 217640 \\
\hline 19 & Cheatoceros danicus & 80 & 0 & $\circ$ & 0 & 0 & 80 \\
\hline 20 & Chaetoceros decipiems & 0 & 0 & 。 & 0 & 160 & 160 \\
\hline 21 & Chaetoceros didymus & 280 & 320 & 。 & 0 & 0 & 600 \\
\hline 22 & Cheatoceros e eibenii & 0 & 。 & 320 & 。 & 0 & 320 \\
\hline 23 & Chaetoceros S Iociniosus & 760 & 160 & 。 & 。 & 0 & 920 \\
\hline 24 & Cheetoceros Ioevis & 0 & 0 & 120 & $\circ$ & 0 & 120 \\
\hline 25 & Chaetoceros lauderi & 0 & 0 & $\circ$ & 0 & 160 & 160 \\
\hline 26 & Cheetoceros Iorenzianus & 8760 & 2000 & ${ }_{1320}$ & 6120 & 7520 & 25720 \\
\hline 27 & chaetocerers pervuianus & 800 & 660 & 400 & 200 & 280 & 2340 \\
\hline 28 & Chaetoceros radicans & 0 & 0 & 360 & 0 & 0 & 360 \\
\hline 29 & Guinardia striata & 6720 & 5960 & 1080 & 1680 & 7400 & 22840 \\
\hline 30 & Choetoceros sp. & 1760 & 1920 & 120 & 480 & 0 & 4280 \\
\hline 31 & Climacodium frouenfeldionum & 240 & 0 & $\circ$ & $\circ$ & 0 & 240 \\
\hline 32 & coscinodiscus perforotus & 0 & 80 & $\circ$ & ${ }_{40}$ & 0 & 120 \\
\hline 33 & Coscinodiscus walesii & 0 & ${ }_{40}$ & $\circ$ & $\circ$ & 0 & 40 \\
\hline 34 & cyclotella sp. & 520 & 40 & $\circ$ & $\circ$ & 0 & 560 \\
\hline 35 & cylindrotheca closterium & 0 & 160 & 160 & 200 & 120 & 640 \\
\hline 36 & Doctyliosolen frogilissimus & 2160 & 520 & 960 & 1080 & 5840 & 10560 \\
\hline 37 & Dactyliosolen phuketensis & 1140 & 40 & 120 & 520 & 0 & 1820 \\
\hline 38 & Detonula pumila & 2160 & 920 & 520 & 880 & 8480 & 12960 \\
\hline 39 & Ditylum brightwellii & 40 & 160 & 120 & $\circ$ & 0 & 320 \\
\hline 40 & Eucampia cornuta & 0 & 0 & 。 & 。 & 480 & 480 \\
\hline 41 & Euccompia zoodiacus & 4160 & 660 & 210 & ${ }_{4320}$ & 2120 & ${ }_{11470}$ \\
\hline 42 & Fragillariopsis doliolus & 320 & $\circ$ & 280 & 。 & 0 & 600 \\
\hline${ }_{43}$ & Grammalophoro marina & 。 & 。 & $\circ$ & o & 120 & 120 \\
\hline 44 & Pseudo-nitzschio delicatissima & 11600 & 23720 & 5840 & 12720 & 10360 & 64240 \\
\hline 45 & Lectocylindrus danicus & 6000 & 3220 & 640 & 4840 & 5160 & 19860 \\
\hline 46 & Guinardia delicktula & 640 & 40 & 0 & 720 & 80 & 1480 \\
\hline 47 & Guinardia floccida & 800 & 520 & 1280 & 960 & 200 & 3760 \\
\hline 48 & Chetoctoros socialis & 12240 & 0 & 6320 & 32640 & 0 & 51200 \\
\hline 49 & Hossea sp. & 840 & 1160 & 520 & 1080 & 1000 & 4600 \\
\hline 50 & Helicotheca thamesis & 0 & 。 & 80 & 0 & 40 & 120 \\
\hline 51 & Hemiaulus houckii & 240 & 80 & 。 & 。 & 200 & 520 \\
\hline 52 & Hemiaulus membranoceus & 320 & 0 & 。 & 0 & 400 & 720 \\
\hline 53 & Hemiaulus sinensis & 320 & 120 & 0 & 0 & 0 & 440 \\
\hline 54 & Lauderia annulato & 720 & 80 & 960 & 920 & 480 & 3160 \\
\hline 55 & Pseudo-nitzschia seriato & 1160 & 240 & 160 & 160 & 80 & 1800 \\
\hline 56 & Leptocyindarus mediterananeus & 680 & 200 & 80 & 440 & 160 & 1560 \\
\hline 57 & Leptocylindrus minimus & 1240 & 280 & 160 & 1560 & 920 & 4160 \\
\hline 58 & Lioloma sp. & 40 & 0 & 0 & 0 & 0 & 40 \\
\hline 59 & Lioloma pacificum & 0 & 40 & $\circ$ & 0 & 0 & 40 \\
\hline 60 & Liomophora sp. & 480 & o & 。 & o & 160 & 640 \\
\hline 61 & Meuniera membrenncea & 3400 & 440 & 120 & 760 & 880 & 5600 \\
\hline 62 & Novicula sp. & 240 & 50 & 40 & 360 & 0 & 690 \\
\hline 63 & Nitzschia longissima & 2440 & 1440 & 720 & 720 & 1000 & 6320 \\
\hline 64 & Nitzschia sp. & 840 & 5680 & 320 & 2160 & 720 & 9720 \\
\hline 65 & Odontella mobiliensis & 120 & $\circ$ & 120 & 0 & 0 & 240 \\
\hline 66 & Odontella sinensis & 0 & 200 & $\circ$ & 0 & 120 & 320 \\
\hline 67 & Pleurssigma sp. & 320 & 240 & 0 & 120 & 40 & 720 \\
\hline 68 & Probossica alota & 440 & 680 & 240 & 360 & so & 1800 \\
\hline 69 & Pseudoguinardia recto & 0 & 0 & $\circ$ & 240 & 80 & 320 \\
\hline 70 & Pseudosolenio calcar-avis & 120 & 160 & 200 & 160 & 80 & 720 \\
\hline 71 & Rhizosolenia bergonil & 0 & 50 & $\circ$ & 0 & 0 & 50 \\
\hline 72 & Rhizosolenia formosa & 0 & 0 & 0 & 80 & 0 & 80 \\
\hline 73 & Rhizosolenia hyadina & 280 & 0 & $\circ$ & 1080 & 0 & 1360 \\
\hline 74 & Rhizesolentic i imbricata & 500 & 0 & $\circ$ & 40 & 0 & 540 \\
\hline 75 & Rhizosolenila pungens & 90 & 160 & $\circ$ & 40 & 120 & 410 \\
\hline 76 & Rhirasolenila setigera & 11600 & 840 & 1760 & 960 & 1360 & 16520 \\
\hline 77 & Rhizosolenia styifformis & 2800 & 1040 & 80 & ${ }_{40}$ & 80 & 4040 \\
\hline 78 & Skeletonema costatum & 3400 & 360 & 1520 & 1200 & 2760 & 9240 \\
\hline 79 & Stephanopopxis turis & 0 & 。 & $\circ$ & 0 & 90 & 90 \\
\hline so & Tholassionema nitzschioides & 2280 & 160 & 320 & 120 & 1120 & 4000 \\
\hline 81 & Thelassiosirira angulata & 0 & 0 & 240 & 。 & 0 & 240 \\
\hline 82 & Tholassiosita sp. & 160 & 80 & 0 & 0 & 1080 & 1320 \\
\hline 83 & Thelassiositra subtibilils & 960 & 80 & 1960 & 960 & 480 & 4440 \\
\hline 84 & Tholassiothrix Iongissima & 0 & 0 & 0 & 120 & 0 & 120 \\
\hline \multirow{2}{*}{85} & Trachyneis aspera & 0 & 140 & 0 & 0 & 0 & 140 \\
\hline & Total general & 146970 & 75780 & 47210 & 129300 & 181730 & 580990 \\
\hline
\end{tabular}


Tabla 3. Composición y abundancia (cel. $\mathrm{L}^{-1}$ ) de Dinoflagelados registrados por estación en el primer trimestre enero marzo 2014. Se resaltan las especies con los valores más altos de abundancia.

\begin{tabular}{|c|c|c|c|c|c|c|c|}
\hline & DINOFLAGELADOS & $\mathrm{E}-1$ & $\mathrm{E}-2$ & $\mathrm{E}-3$ & E-4 & E-5 & TOTAL \\
\hline 1 & Amphidinium sp. & 0 & 0 & 40 & 0 & 0 & 40 \\
\hline 2 & Ceratium massiliense & 40 & 0 & 0 & 0 & 40 & 80 \\
\hline 3 & Ceratium furca & 0 & 40 & 0 & 0 & 80 & 120 \\
\hline 4 & Ceratium breve & 440 & 280 & 320 & 240 & 280 & 1560 \\
\hline 5 & Ceratium kofoidii & 0 & 0 & 0 & 40 & 40 & 80 \\
\hline 6 & Ceratium macroceros & 0 & 0 & 0 & 40 & 0 & 40 \\
\hline 7 & Ceratium trichoceros & 40 & 0 & 0 & 0 & 0 & 40 \\
\hline 8 & Ceratium tripos & 0 & 0 & 160 & 0 & 0 & 160 \\
\hline 9 & Dinophysis caudata & 0 & 0 & 40 & 0 & 0 & 40 \\
\hline 10 & Diplopelta asymmetrica & 0 & 0 & 0 & 40 & 0 & 40 \\
\hline 11 & Gymnodinium sp. & 0 & 0 & 120 & 1360 & 160 & 1640 \\
\hline 12 & Gyrodinium sp. & 560 & 1640 & 40 & 4080 & 2200 & 8520 \\
\hline 13 & Noctiluca scintillans & 80 & 0 & 0 & 40 & 0 & 120 \\
\hline 14 & Peridinium quinquecorne & 40 & 0 & 0 & 0 & 0 & 40 \\
\hline 15 & Polykrikos sp. & 0 & 0 & 0 & 0 & 80 & 80 \\
\hline 16 & Pronoctiluca sp. & 40 & 40 & 40 & 40 & 80 & 240 \\
\hline 17 & Prorocentrum gracile & 0 & 0 & 0 & 40 & 0 & 40 \\
\hline 18 & Prorocentrum micans & 3320 & 120 & 200 & 80 & 0 & 3720 \\
\hline 19 & Prorocentrum sp. & 360 & 80 & 0 & 40 & 0 & 480 \\
\hline 20 & Protoperidinium depressum & 360 & 120 & 80 & 160 & 200 & 920 \\
\hline 21 & Protoperidinium minutum & 0 & 40 & 0 & 0 & 0 & 40 \\
\hline 22 & Protoperidinium pellucidum & 0 & 40 & 0 & 0 & 0 & 40 \\
\hline 23 & Protoperidinium sp. & 40 & 0 & 0 & 40 & 0 & 80 \\
\hline 24 & Pyrophacus horologium & 0 & 80 & 0 & 0 & 0 & 80 \\
\hline \multirow[t]{2}{*}{25} & Scrippsiella trochoidea & 80 & 320 & 280 & 120 & 40 & 840 \\
\hline & Total general & 5400 & 2800 & 1320 & 6360 & 3200 & 19080 \\
\hline
\end{tabular}


Tabla 4. Composición y abundancia $\left(\mathrm{cel} \cdot \mathrm{L}^{-1}\right)$ de Diatomeas registradas por estación en el segundo trimestre abril - junio 2014.

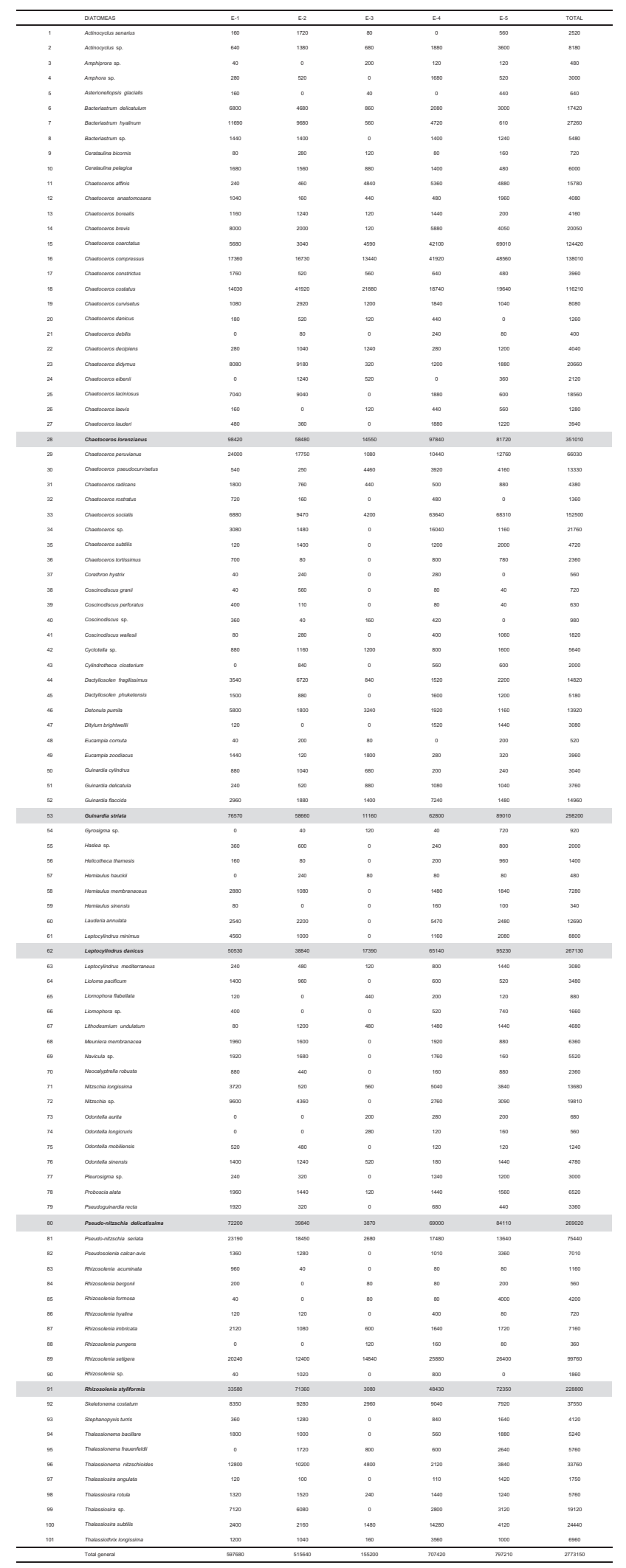

Se resaltan las especies con los valores mas altos de abundancia. 
Tabla 5. Composición y abundancia (cel-L-1) de Dinoflagelados registrados por estación en el segundo trimestre abril - junio 2014.

\begin{tabular}{|c|c|c|c|c|c|c|c|}
\hline & DINOFLAGELADOS & $\mathrm{E}-1$ & $\mathrm{E}-2$ & $E-3$ & $\overline{E-4}$ & $\mathrm{E}-5$ & TOTAL \\
\hline 1 & Alexandrium sp. & 280 & 120 & 0 & 0 & 200 & 600 \\
\hline 2 & Amphidinium sp. & 0 & 0 & 0 & 40 & 0 & 40 \\
\hline 3 & Ceratium breve & 0 & 40 & 1120 & 1240 & 520 & 2920 \\
\hline 4 & Ceratium falcatum & 40 & 40 & 680 & 0 & 80 & 840 \\
\hline 5 & Ceratium furca & 440 & 2440 & 1200 & 1480 & 2760 & 8320 \\
\hline 6 & Ceratium fusus var fusus & 0 & 0 & 0 & 80 & 0 & 80 \\
\hline 7 & Ceratium fusus var. furca & 0 & 0 & 40 & 160 & 0 & 200 \\
\hline 8 & Ceratium kofoidii & 0 & 1600 & 80 & 0 & 0 & 1680 \\
\hline 9 & Ceratium trichoceros & 40 & 0 & 0 & 0 & 0 & 40 \\
\hline 10 & Corythodinium sp. & 40 & 480 & 0 & 0 & 0 & 520 \\
\hline 11 & Dinophysis caudata & 0 & 0 & 280 & 480 & 40 & 800 \\
\hline 12 & Dinophysys rotundala & 0 & 0 & 0 & 40 & 40 & 80 \\
\hline 13 & Diplopelta asymetrica & 40 & 0 & 0 & 40 & 0 & 80 \\
\hline 14 & Diplopeltopsis minor & 80 & 0 & 0 & 120 & 80 & 280 \\
\hline 15 & Diplopsalis sp. & 0 & 880 & 40 & 40 & 0 & 960 \\
\hline 16 & Gonyaulax sp. & 160 & 360 & 160 & 40 & 520 & 1240 \\
\hline 17 & Gymnodinium sp. & 840 & 0 & 0 & 480 & 400 & 1720 \\
\hline 18 & Gyrodinium sp. & 160 & 40 & 800 & 4440 & 400 & 5840 \\
\hline 19 & Lingulodinium polyedrum & 0 & 0 & 40 & 200 & 40 & 280 \\
\hline 20 & Nocticula sp & 640 & 40 & 0 & 0 & 0 & 680 \\
\hline 21 & Noctiluca scintillans & 680 & 280 & 80 & 0 & 160 & 1200 \\
\hline 22 & Oxytoxum sp. & 40 & 40 & 40 & 0 & 120 & 240 \\
\hline 23 & Pronoctiluca sp. & 1200 & 300 & 720 & 0 & 120 & 2340 \\
\hline 24 & Prorocentrum dentatum & 40 & 0 & 0 & 0 & 0 & 40 \\
\hline 25 & Prorocentrum gracile & 80 & 0 & 0 & 0 & 0 & 80 \\
\hline 26 & Prorocentrum gradie & 240 & 0 & 0 & 0 & 0 & 240 \\
\hline 27 & Prorocentrum micans & 2680 & 720 & 80 & 360 & 240 & 4080 \\
\hline 28 & Prorocentrum minimum & 0 & 0 & 40 & 40 & 40 & 120 \\
\hline 29 & Prorocentrum sigmoides & 0 & 0 & 0 & 0 & 40 & 40 \\
\hline 30 & Prorocentrum sp. & 80 & 0 & 0 & 80 & 80 & 240 \\
\hline 31 & Protoperidinium bispinum & 40 & 0 & 0 & 40 & 80 & 160 \\
\hline 32 & Protoperidinium conicum & 0 & 0 & 40 & 0 & 0 & 40 \\
\hline 33 & Protoperidinium crassipes & 40 & 40 & 420 & 120 & 120 & 740 \\
\hline 34 & Protoperidinium depresssum & 0 & 40 & 0 & 40 & 0 & 80 \\
\hline 35 & Protoperidinium divergens & 120 & 40 & 0 & 0 & 0 & 160 \\
\hline 36 & Protoperidinium latispinum & 0 & 0 & 0 & 120 & 0 & 120 \\
\hline 37 & Protoperidinium minutissimum & 0 & 0 & 80 & 0 & 0 & 80 \\
\hline 38 & Protoperidinium minutum & 0 & 0 & 80 & 0 & 40 & 120 \\
\hline 39 & Protoperidinium ovum & 80 & 40 & 760 & 80 & 0 & 960 \\
\hline 40 & Protoperidinium pellucidum & 80 & 80 & 280 & 440 & 120 & 1000 \\
\hline 41 & Protoperidinium pentagonum & 0 & 0 & 40 & 80 & 0 & 120 \\
\hline 42 & Protoperidinium quarnerense & 0 & 0 & 0 & 40 & 0 & 40 \\
\hline 43 & Protoperidinium sp. & 560 & 360 & 460 & 0 & 280 & 1660 \\
\hline 44 & Protoperidinium steinii & 40 & 80 & 40 & 40 & 0 & 200 \\
\hline 45 & Protoperidinium tuba & 40 & 120 & 0 & 120 & 400 & 680 \\
\hline 46 & Protoperidinium vulgare & 0 & 40 & 0 & 0 & 0 & 40 \\
\hline 47 & Pyrophacus horologium & 80 & 40 & 80 & 0 & 80 & 280 \\
\hline 48 & Pyrophacus steinii & 0 & 0 & 40 & 40 & 0 & 80 \\
\hline 49 & Scrippsiella spinifera & 6480 & 2000 & 40 & 0 & 80 & 8600 \\
\hline \multirow[t]{2}{*}{50} & Scrippsiella trochoidea & 440 & 40 & 120 & 18920 & 880 & 20400 \\
\hline & Total general & 15800 & 10300 & 7880 & 29440 & 7960 & 71380 \\
\hline
\end{tabular}

Se resaltan las especies co.n los valores mas altos de abundancia. 
Las variables ambientales se registraron de la siguiente manera: La temperatura superficial del mar osciló entre $22,4^{\circ} \mathrm{C}$ a $29^{\circ} \mathrm{C}$; la salinidad entre 30,1 UPS y 33,4 UPS; el pH entre 8 a 8,2 y el oxígeno disuelto entre 6,1 a 7,1 $\mathrm{mg} \cdot \mathrm{L}^{-1}$, valores que se encuentran dentro de Estándar de Calidad Ambiental de agua marina (MINAM, 2008).

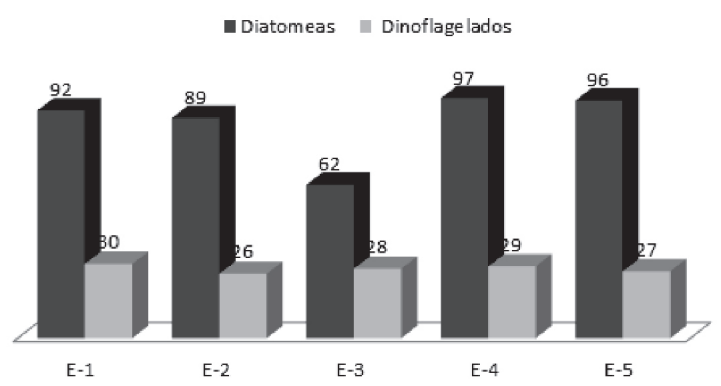

Figura 6. Riqueza del Fitoplancton registrado en el segundo trimestre en las estaciones de muestreo. E-1 = A $500 \mathrm{~m}$ al noreste de la Plataforma, a favor de la corriente; $\mathrm{E}-2=\mathrm{A} 500 \mathrm{~m}$ al noroeste de la Plataforma, en contra de la corriente; E-3= Plataforma Albacora; E-4=2 Km, al suroeste de la Plataforma; E-5 = $2 \mathrm{Km}$, al sureste de la Plataforma.

Diatomeas Dinoflagelados

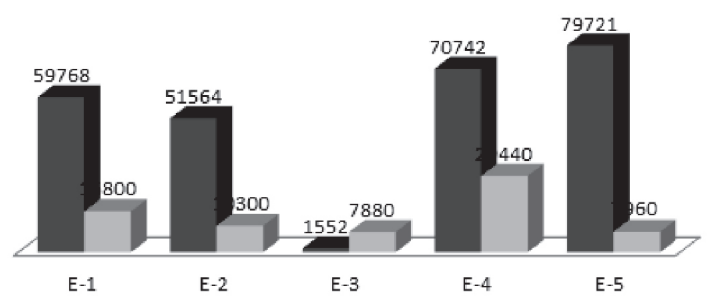

Figura 7. Abundancia (cel. $\left.\mathrm{L}^{-1}\right)$ del Fitoplancton registrado en el segundo trimestre en las estaciones de muestreo. $\mathrm{E}-1=$ A $500 \mathrm{~m}$ al noreste de la Plataforma, a favor de la corriente; E-2= A $500 \mathrm{~m}$ al noroeste de la Plataforma, en contra de la corriente; E-3= Plataforma Albacora; E-4= 2 $\mathrm{Km}$, al suroeste de la Plataforma; E- $5=2 \mathrm{Km}$, al sureste de la Plataforma.

En el tercer trimestre (julio-setiembre 2014) la riqueza del fitoplancton marino evaluado fue de 107 especies y la abundancia de $283.150 \mathrm{cel} \cdot \mathrm{L}^{-1}$ entre diatomeas y dinoflagelados. Las diatomeas dominaron con el género Chaetoceros coincidiendo con Ochoa \& Gómez (1997), con la especie más abundante Chaetoceros socialis H.S.Lauder, 1864 con $37.440 \mathrm{cel} \cdot \mathrm{L}^{-1}$ (Tabla 6). Las estaciones con mayor número de especies e individuos fueron E-4 y E-5, en cuanto la estación E-3 presentó mayor abundancia (Figura 9). Los dinoflagelados se presentó con la especie más abundante Prorocentrum micans Ehrenberg, 1834 con 3,720 cel.L $\mathrm{L}^{-1}$ (Tabla 7), coincidiendo con Villanueva (1997) en el mes de agosto. La estación con mayor riqueza y abundancia fue E-5 (Figuras 8-9). Las variables ambientales se registraron de la siguiente manera: La temperatura superficial del mar osciló entre $23,8^{\circ} \mathrm{C}$ a $26,3^{\circ} \mathrm{C}$; la salinidad entre 27,4 a 34 UPS; el pH entre 8 a 8,2 y el oxígeno disuelto entre 6,4 y 7,6 mg. $\mathrm{L}^{-1}$, encontrándose dentro de los valores del ECA para agua (MINAM, 2008).

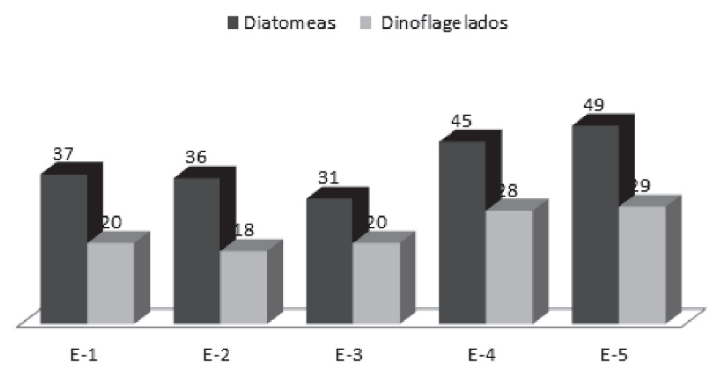

Figura 8. Riqueza del Fitoplancton registrado en el tercer trimestre en las estaciones de muestreo. E-1= A $500 \mathrm{~m}$ al noreste de la Plataforma, a favor de la corriente; E-2= A $500 \mathrm{~m}$ al noroeste de la Plataforma, en contra de la corriente; E-3= Plataforma Albacora; E4= $2 \mathrm{Km}$, al suroeste de la Plataforma; E-5 $=2 \mathrm{Km}$, al sureste de la Plataforma.

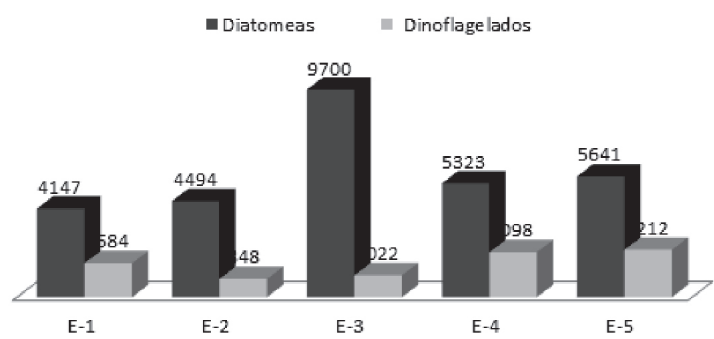

Figura 9. Abundancia $\left(\mathrm{cel} \cdot \mathrm{L}^{-1}\right)$ del Fitoplancton registrado en el tercer trimestre en las estaciones de muestreo. $\mathrm{E}-1=\mathrm{A} 500 \mathrm{~m}$ al noreste de la Plataforma, a favor de la corriente; E-2= A $500 \mathrm{~m}$ al noroeste de la Plataforma, en contra de la corriente; E-3= Plataforma Albacora; E-4= 2 $\mathrm{Km}$, al suroeste de la Plataforma; E-5 $=2 \mathrm{Km}$, al sureste de la Plataforma.

En el cuarto trimestre (octubre-diciembre 2014) las diatomeas y los dinoflagelados 44 registraron un total de 134 especies y la abundancia de $942.430 \mathrm{cel} \cdot \mathrm{L}^{-1}$ entre diatomeas y dinoflagelados. Las diatomeas se registraron con el dominio del genero Chaetoceros con 13 especies, siendo la especie más abundante $C$. socialis con 37,440 cel. $\mathrm{L}^{-1}$ (Tabla 8). Y los dinoflagelados registraron al género Prorocentrum con 3 especies, con su especie más abundante $P$. micans con $3,720 \mathrm{cel} \cdot \mathrm{L}^{-1}$ (Tabla 9). 
Tabla 6. Composición y abundancia (cel. $\left.\mathrm{L}^{-1}\right)$ de Diatomeas registradas por estación en el tercer trimestre julio - setiembre 2014.

\begin{tabular}{|c|c|c|c|c|c|c|c|}
\hline & DIATOMEAS & $\mathrm{E}-1$ & $\mathrm{E}-2$ & $\mathrm{E}-3$ & $\mathrm{E}-4$ & $E-5$ & Total \\
\hline 1 & Amphiprora sp. & 0 & 0 & 0 & 40 & 240 & 280 \\
\hline 2 & Amphora sp. & 0 & 0 & 0 & 400 & 240 & 640 \\
\hline 3 & Bacteriastrum delicatulum & 40 & 0 & 40 & 0 & 0 & 80 \\
\hline 4 & Bacteriastrum hyalinum & 280 & 0 & 0 & 280 & 0 & 560 \\
\hline 5 & Bacteriastrum sp. & 960 & 320 & 0 & 200 & 80 & 1560 \\
\hline 6 & Cerataulina bicornis & 0 & 400 & 0 & 200 & 280 & 880 \\
\hline 7 & Cerataulina pelagica & 0 & 240 & 0 & 140 & 260 & 640 \\
\hline 8 & Chaetoceros anastomosans & 80 & 40 & 0 & 0 & 0 & 120 \\
\hline 9 & Chaetoceros brevis & 40 & 240 & 40 & 160 & 160 & 640 \\
\hline 10 & Chaetoceros compressus & 0 & 1080 & 780 & 2720 & 2840 & 7420 \\
\hline 11 & Chaetoceros costatus & 800 & 720 & 0 & 740 & 120 & 2380 \\
\hline 12 & Chaetoceros curvisetus & 1020 & 0 & 0 & 1800 & 2000 & 4820 \\
\hline 13 & Chaetoceros decipiens & 0 & 330 & 0 & 560 & 120 & 1010 \\
\hline 14 & Chaetoceros didymus & 0 & 0 & 160 & 0 & 0 & 160 \\
\hline 15 & Chaetoceros laciniosus & 160 & 0 & 0 & 0 & 0 & 160 \\
\hline 16 & Chaetoceros lauderi & 0 & 200 & 0 & 0 & 0 & 200 \\
\hline 17 & Chaetoceros Iorenzianus & 4800 & 3600 & 240 & 4080 & 4060 & 16780 \\
\hline 18 & Chaetoceros peruvianus & 0 & 80 & 0 & 440 & 0 & 520 \\
\hline 19 & Chaetoceros socialis & 6960 & 10440 & 1440 & 9720 & 8880 & 37440 \\
\hline 20 & Chaetoceros sp. & 80 & 200 & 0 & 0 & 440 & 720 \\
\hline 21 & Corethron hystrix & 0 & 0 & 40 & 0 & 0 & 40 \\
\hline 22 & Coscinodiscus sp. & 0 & 0 & 0 & 120 & 40 & 160 \\
\hline 23 & Coscinodiscus wailesii & 0 & 0 & 40 & 0 & 160 & 200 \\
\hline 24 & Cyclotella sp. & 40 & 0 & 0 & 120 & 0 & 160 \\
\hline 25 & Dactyliosolen fragilisssimus & 0 & 0 & 0 & 760 & 520 & 1280 \\
\hline 26 & Dactyliosolen phuketensis & 0 & 240 & 0 & 0 & 680 & 920 \\
\hline 27 & Detonula pumila & 0 & 360 & 0 & 0 & 640 & 1000 \\
\hline 28 & Ditylum brightwellii & 0 & 0 & 240 & 0 & 160 & 400 \\
\hline 29 & Eucampia cornuta & 0 & 0 & 100 & 80 & 0 & 180 \\
\hline 30 & Guinardia delicatula & 0 & 0 & 0 & 160 & 0 & 160 \\
\hline 31 & Guinardia flaccida & 960 & 0 & 100 & 1040 & 120 & 2220 \\
\hline 32 & Guinardia striata & 4480 & 4000 & 1280 & 10840 & 10960 & 31560 \\
\hline 33 & Haslea sp. & 200 & 560 & 0 & 0 & 160 & 920 \\
\hline 34 & Helicotheca thamesis & 0 & 0 & 0 & 40 & 40 & 80 \\
\hline 35 & Hemiaulus hauckii & 0 & 0 & 0 & 0 & 40 & 40 \\
\hline 36 & Hemiaulus membranaceus & 0 & 80 & 40 & 0 & 0 & 120 \\
\hline 37 & Lauderia annulata & 0 & 120 & 40 & 0 & 20 & 180 \\
\hline 38 & Leptocylindrus danicus & 7320 & 7280 & 1060 & 2220 & 4000 & 21880 \\
\hline 39 & Leptocylindrus mediterraneus & 0 & 120 & 600 & 160 & 0 & 880 \\
\hline 40 & Leptocylindrus minimus & 280 & 0 & 80 & 80 & 480 & 920 \\
\hline 41 & Lioloma pacificum & 1080 & 1140 & 640 & 1080 & 1000 & 4940 \\
\hline 42 & Lioloma sp. & 0 & 120 & 0 & 0 & 0 & 120 \\
\hline 43 & Lithodesmium undulatum & 40 & 0 & 0 & 80 & 0 & 120 \\
\hline 44 & Meuniera membranacea & 0 & 0 & 0 & 80 & 110 & 190 \\
\hline 45 & Neocalyptrella robusta & 490 & 0 & 80 & 120 & 400 & 1090 \\
\hline 46 & Nitzschia longissima & 0 & 0 & 0 & 40 & 120 & 160 \\
\hline 47 & Nitzschia sp. & 320 & 0 & 0 & 0 & 720 & 1040 \\
\hline 48 & Pleurosigma sp. & 240 & 0 & 0 & 40 & 620 & 900 \\
\hline 49 & Proboscia alata & 0 & 120 & 160 & 40 & 0 & 320 \\
\hline 50 & Pseudoguinardia recta & 120 & 480 & 80 & 360 & 360 & 1400 \\
\hline 51 & Pseudo-nitzschia delicatissima & 2680 & 6000 & 1120 & 10080 & 10020 & 29900 \\
\hline 52 & Pseudo-nitzschia seriata & 480 & 200 & 240 & 760 & 0 & 1680 \\
\hline 53 & Pseudosolenia calcar-avis & 160 & 0 & 0 & 120 & 520 & 800 \\
\hline 54 & Rhizosolenia bergonii & 160 & 40 & 0 & 80 & 120 & 400 \\
\hline 55 & Rhizosolenia hyalina & 40 & 40 & 40 & 0 & 40 & 160 \\
\hline 56 & Rhizosolenia imbricata & 120 & 160 & 0 & 160 & 160 & 600 \\
\hline 57 & Rhizosolenia pungens & 0 & 80 & 160 & 80 & 0 & 320 \\
\hline 58 & Rhizosolenia setigera & 80 & 0 & 20 & 0 & 80 & 180 \\
\hline 59 & Rhizosolenia styliformis & 4880 & 4240 & 80 & 2000 & 2080 & 13280 \\
\hline 60 & Skeletonema costatum & 680 & 0 & 0 & 0 & 560 & 1240 \\
\hline 61 & Stephanopyxis turris & 0 & 150 & 0 & 0 & 80 & 230 \\
\hline 62 & Thalassionema frauenfeldii & 240 & 880 & 640 & 130 & 0 & 1890 \\
\hline 63 & Thalassionema nitzschioides & 40 & 0 & 20 & 0 & 80 & 140 \\
\hline 64 & Thalassiosira rotula & 880 & 0 & 0 & 120 & 680 & 1680 \\
\hline 65 & Thalassiosira sp. & 0 & 520 & 0 & 520 & 0 & 1040 \\
\hline 66 & Thalassiosira subtilis & 0 & 120 & 80 & 160 & 480 & 840 \\
\hline 67 & Thalassiothrix Iongissima & 120 & 0 & 0 & 0 & 280 & 400 \\
\hline 68 & Trachyneis sp. & 0 & 0 & 20 & 80 & 40 & 140 \\
\hline \multirow[t]{2}{*}{69} & Trachyneis sp. & 120 & 0 & 0 & 0 & 120 & 240 \\
\hline & Total general & 41470 & 44940 & 9700 & 53230 & 56410 & 205750 \\
\hline
\end{tabular}

Se resaltan las especies con los valores mas altos de abundancia. 
Tabla 7. Composición y abundancia $\left(\mathrm{cel} \cdot \mathrm{L}^{-1}\right)$ de Dinoflagelados registrados por estación en el tercer trimestre Julio setiembre 2014.

\begin{tabular}{|c|c|c|c|c|c|c|c|}
\hline & DINOFLAGELADOS & $\mathrm{E}-1$ & $\mathrm{E}-2$ & $E-3$ & $\mathrm{E}-4$ & E-5 & TOTAL \\
\hline 1 & Alexandrium sp. & 40 & 0 & 2000 & 80 & 0 & 2120 \\
\hline 2 & Amphidinium sp. & 240 & 0 & 40 & 0 & 0 & 280 \\
\hline 3 & Ceratium breve & 1040 & 1720 & 90 & 1060 & 4880 & 8790 \\
\hline 4 & Ceratium dens & 0 & 0 & 0 & 0 & 80 & 80 \\
\hline 5 & Ceratium furca & 140 & 40 & 300 & 7440 & 3570 & 11490 \\
\hline 6 & Ceratium kofoidii & 80 & 0 & 40 & 200 & 200 & 520 \\
\hline 7 & Ceratium trichoceros & 0 & 0 & 0 & 40 & 40 & 80 \\
\hline 8 & Dinophysis caudata & 0 & 0 & 0 & 320 & 120 & 440 \\
\hline 9 & Diplopelta asymmetrica & 0 & 0 & 40 & 40 & 40 & 120 \\
\hline 10 & Diplopelta cf. globulus & 40 & 0 & 0 & 0 & 0 & 40 \\
\hline 11 & Diplopeltopsis minor & 0 & 0 & 0 & 80 & 0 & 80 \\
\hline 12 & Goniodoma polyedricum & 800 & 780 & 30 & 200 & 80 & 1890 \\
\hline 13 & Gonyaulax polyedra & 1240 & 1600 & 160 & 120 & 220 & 3340 \\
\hline 14 & Gymnodinium sp. & 520 & 400 & 100 & 5080 & 4920 & 11020 \\
\hline 15 & Gyrodinium sp. & 2160 & 1640 & 600 & 3240 & 5360 & 13000 \\
\hline 16 & Lingulodinium polyedrum & 120 & 240 & 40 & 160 & 160 & 720 \\
\hline 17 & Noctiluca scintillans & 80 & 0 & 0 & 280 & 50 & 410 \\
\hline 18 & Ornithocercus orbiculatus & 0 & 0 & 40 & 40 & 40 & 120 \\
\hline 19 & Oxytoxum sp. & 1440 & 1040 & 920 & 440 & 480 & 4320 \\
\hline 20 & Peridinium quinquecorne & 0 & 0 & 0 & 40 & 0 & 40 \\
\hline 21 & Phalacroma rotundatum & 100 & 280 & 80 & 40 & 40 & 540 \\
\hline 22 & Podopalma bipes & 0 & 200 & 120 & 400 & 40 & 760 \\
\hline 23 & Pronoctiluca sp. & 0 & 40 & 200 & 160 & 600 & 1000 \\
\hline 24 & Prorocentrum gracile & 40 & 0 & 0 & 0 & 0 & 40 \\
\hline 25 & Prorocentrum micans & 7360 & 40 & 4880 & 400 & 280 & 12960 \\
\hline 26 & Prorocentrum minimun & 40 & 40 & 0 & 0 & 40 & 120 \\
\hline 27 & Prorocentrum sigmoides & 0 & 0 & 0 & 40 & 40 & 80 \\
\hline 28 & Prorocentrum sp. & 0 & 200 & 0 & 120 & 40 & 360 \\
\hline 29 & Protoperidinium cf. bipes & 0 & 40 & 0 & 0 & 0 & 40 \\
\hline 30 & Protoperidinium cf. tuba & 40 & 0 & 40 & 120 & 120 & 320 \\
\hline 31 & Protoperidinium crassipes & 0 & 0 & 40 & 0 & 40 & 80 \\
\hline 32 & Protoperidinium excentricum & 0 & 0 & 0 & 0 & 40 & 40 \\
\hline 33 & Protoperidinium latispinum & 0 & 0 & 0 & 0 & 40 & 40 \\
\hline 34 & Protoperidinium leonis & 0 & 40 & 0 & 40 & 0 & 80 \\
\hline 35 & Protoperidinium mendiolae & 0 & 0 & 0 & 40 & 0 & 40 \\
\hline 36 & Protoperidinium sp. & 160 & 40 & 80 & 160 & 200 & 640 \\
\hline 37 & Pyrophacus horologium & 0 & 0 & 0 & 0 & 40 & 40 \\
\hline \multirow[t]{2}{*}{38} & Scrippsiella trochoidea & 160 & 100 & 140 & 600 & 320 & 1320 \\
\hline & Total general & 15840 & 8480 & 9980 & 20980 & 22120 & 77400 \\
\hline
\end{tabular}

Se resaltan las especies con los valores mas altos de abundancia. 
Tabla 8. Composición y abundancia $\left(\mathrm{cel} \cdot \mathrm{L}^{-1}\right)$ de Diatomeas registradas por estación en el cuarto trimestre octubre Diciembre 2014.

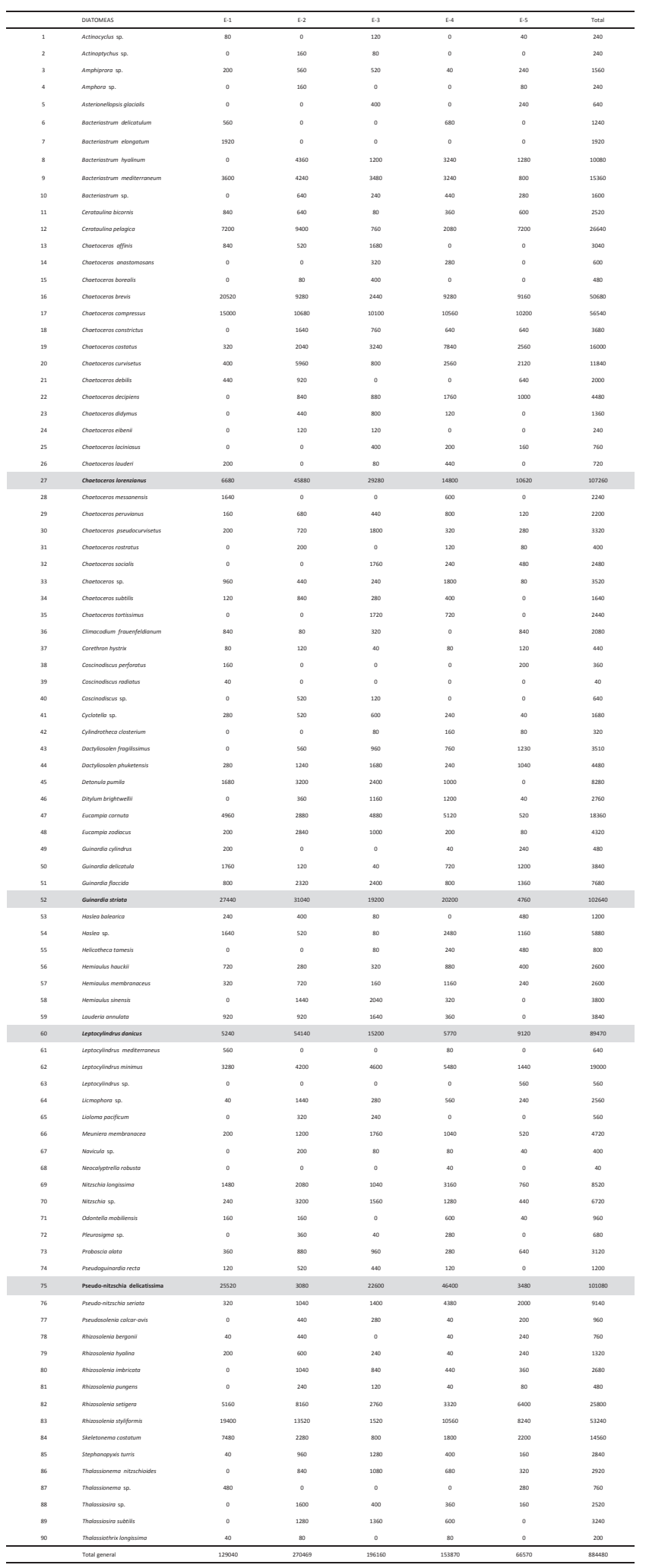

Se resaltan las especies con los valores mas altos de abundancia. 
Tabla 9. Composición y abundancia (cel. $\left.\mathrm{L}^{-1}\right)$ de Dinoflagelados registrados por estación en el cuarto trimestre octubre - Diciembre 2014.

\begin{tabular}{|c|c|c|c|c|c|c|c|}
\hline & DINOFLAGELADOS & $\mathrm{E}-1$ & $\mathrm{E}-2$ & $E-3$ & $E-4$ & $\mathrm{E}-5$ & Total \\
\hline 1 & Alexandrium sp. & 160 & 160 & 1720 & 140 & 280 & 2460 \\
\hline 2 & Alexandrium tamiyavanichii & 440 & 680 & 0 & 600 & 360 & 2080 \\
\hline 3 & Amphidinium sp. & 0 & 0 & 0 & 80 & 0 & 80 \\
\hline 4 & Blepharocysta sp. & 0 & 0 & 40 & 0 & 0 & 40 \\
\hline 5 & Ceratium breve & 40 & 120 & 0 & 560 & 1600 & 2320 \\
\hline 6 & Ceratium furca & 4520 & 1600 & 40 & 1240 & 2220 & 9620 \\
\hline 7 & Ceratium kofoidii & 40 & 160 & 0 & 40 & 120 & 360 \\
\hline 8 & Ceratium massiliense & 0 & 40 & 0 & 80 & 160 & 280 \\
\hline 9 & Corythodinium sp. & 0 & 40 & 0 & 80 & 80 & 200 \\
\hline 10 & Dinophysis exigua & 0 & 40 & 0 & 420 & 80 & 540 \\
\hline 11 & Diplopelta asymmetrica & 80 & 0 & 40 & 0 & 0 & 120 \\
\hline 12 & Diplopeltopsis minor & 0 & 0 & 40 & 40 & 80 & 160 \\
\hline 13 & Dynophysis caudata & 160 & 160 & 80 & 0 & 40 & 440 \\
\hline 14 & Goniodoma polyedricum & 40 & 0 & 940 & 280 & 520 & 1780 \\
\hline 15 & Gonyaulax polyedra & 1600 & 1800 & 0 & 360 & 800 & 4560 \\
\hline 16 & Gonyaulax sp. & 200 & 480 & 0 & 760 & 320 & 1760 \\
\hline 17 & Gymnodinium sp. & 800 & 600 & 880 & 2400 & 1480 & 6160 \\
\hline 18 & Gyrodinium falcatum & 40 & 40 & 40 & 80 & 40 & 240 \\
\hline 19 & Gyrodinium sp. & 2120 & 2000 & 120 & 1320 & 1680 & 7240 \\
\hline 20 & Kofoidinium sp. & 0 & 0 & 0 & 40 & 40 & 80 \\
\hline 21 & Noctiluca scintillans & 0 & 40 & 40 & 0 & 40 & 120 \\
\hline 22 & Ornithocercus orbiculatus & 80 & 80 & 0 & 560 & 200 & 920 \\
\hline 23 & Oxytoxum scolopax & 0 & 40 & 0 & 80 & 40 & 160 \\
\hline 24 & Oxytoxum sp. & 80 & 120 & 0 & 160 & 320 & 680 \\
\hline 25 & Podolampas bipes & 80 & 160 & 0 & 280 & 0 & 520 \\
\hline 26 & Podolampas palmipes & 0 & 40 & 0 & 0 & 0 & 40 \\
\hline 27 & Pronoctiluca sp. & 0 & 0 & 40 & 40 & 120 & 200 \\
\hline 28 & Prorocentrum compressum & 80 & 0 & 0 & 0 & 560 & 640 \\
\hline 29 & Prorocentrum micans & 2800 & 1720 & 160 & 2040 & 1280 & 8000 \\
\hline 30 & Prorocentrum sp. & 0 & 40 & 0 & 160 & 80 & 280 \\
\hline 31 & Prorocentrum triestinum & 40 & 0 & 0 & 580 & 0 & 620 \\
\hline 32 & Protoperidinium bipes & 40 & 40 & 0 & 0 & 0 & 80 \\
\hline 33 & Protoperidinium cassum & 40 & 0 & 0 & 40 & 40 & 120 \\
\hline 34 & Protoperidinium cf. bispinum & 0 & 80 & 0 & 40 & 0 & 120 \\
\hline 35 & Protoperidinium crassipes & 0 & 0 & 80 & 0 & 40 & 120 \\
\hline 36 & Protoperidinium granii & 40 & 40 & 0 & 800 & 0 & 880 \\
\hline 37 & Protoperidinium minutum & 40 & 0 & 0 & 0 & 40 & 80 \\
\hline 38 & Protoperidinium ovum & 160 & 0 & 0 & 160 & 400 & 720 \\
\hline 39 & Protoperidinium pellucidum & 120 & 0 & 80 & 200 & 0 & 400 \\
\hline 40 & Protoperidinium quarnerense & 80 & 80 & 0 & 0 & 40 & 200 \\
\hline 41 & Protoperidinium sp. & 80 & 80 & 0 & 800 & 40 & 1000 \\
\hline 42 & Pyrophacus horologium & 0 & 0 & 0 & 80 & 0 & 80 \\
\hline 43 & Scrippsiella trochoidea & 120 & 480 & 0 & 200 & 400 & 1200 \\
\hline \multirow[t]{2}{*}{44} & Torodinium sp. & 40 & 0 & 40 & 120 & 120 & 320 \\
\hline & Total general & 8560 & 11600 & 10100 & 5600 & 10660 & 58020 \\
\hline
\end{tabular}

Se resaltan las especies con los valores mas altos de abundancia. 
En cuanto a las variables ambientales se registraron de la siguiente manera: La temperatura superficial del mar osciló entre $23,9^{\circ} \mathrm{C}$ y $26,2^{\circ} \mathrm{C}$ similar al trimestre anterior; la salinidad entre 32 a 34 UPS; el pH se mantuvo igual al trimestre anterior y el oxígeno disuelto entre 6,8 a 7,4 $\mathrm{mg} \cdot \mathrm{L}^{-1}$, encontrándose dentro de los valores del ECA para agua marina (MINAM, 2008).

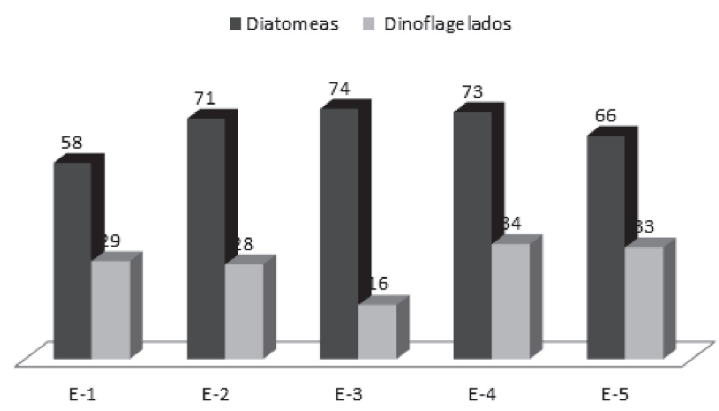

Figura 10. Riqueza del Fitoplancton registrado en el cuarto trimestre en las estaciones de muestreo. E-1= A $500 \mathrm{~m}$ al noreste de la Plataforma, a favor de la corriente; $\mathrm{E}-2=\mathrm{A} 500 \mathrm{~m}$ al noroeste de la Plataforma, en contra de la corriente; E-3= Plataforma Albacora; E-4= $2 \mathrm{Km}$, al suroeste de la Plataforma; E-5 $=2 \mathrm{Km}$, al sureste de la Plataforma.

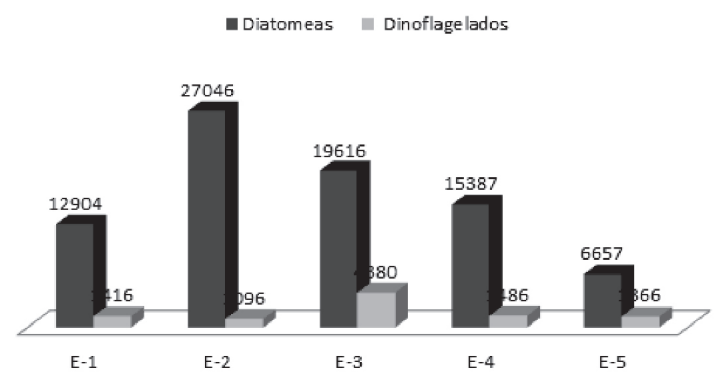

Figura 11. Abundancia (cel. $\left.\mathrm{L}^{-1}\right)$ del Fitoplancton registrado en el cuarto trimestre en las estaciones de muestreo. E-1= A $500 \mathrm{~m}$ al noreste de la Plataforma, a favor de la corriente; $\mathrm{E}-2=\mathrm{A} 500 \mathrm{~m}$ al noroeste de la Plataforma, en contra de la corriente; E-3= Plataforma Albacora; E-4= $2 \mathrm{Km}$, al suroeste de la Plataforma; E-5= $2 \mathrm{Km}$, al sureste de la Plataforma.

\section{Índices de diversidad alfa}

Los índices de diversidad del fitoplancton marino durante el año 2014 (Fig. 12) reflejaron valores variables en todas las estaciones. El índice de Shannon-Wiener presentó valores similares en las estaciones E-1, E-2 y E-3. Se presenta un valor promedio mostrando la diversidad media entre la riqueza y la abundancia en el área evaluada. El índice de Simpson muestra también valores similares entre las estaciones E-1, E-2 y E-3, entre 0,91 a 0,95, mostrando que los individuos seleccionados aleatoriamente sean de especies diferentes. El índice de Pielou registró valores similares en las tres primeras estaciones, valores que están entre 0,51 a 0,70 correspondientes a una situación donde las especies son distribuidas de manera equitativa en las estaciones evaluadas, encontrándose un ambiente favorable, concordando con lo señalado por Alcolado (2001).

Se muestra en la Figura 13, la abundancia relativa (\%) del fitoplancton observado con mayor frecuencia y registrado en los meses de enero a diciembre del ańo 2014.

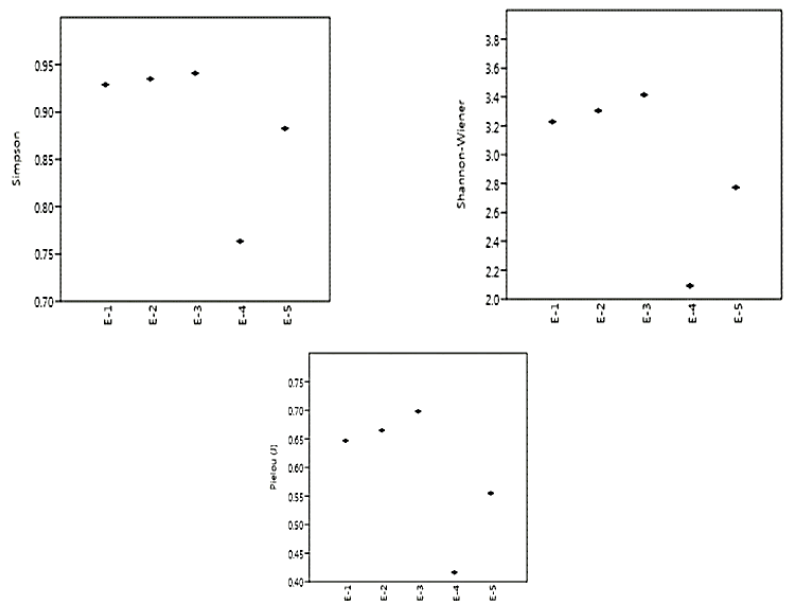

Figura 12. Evaluación de los Índices de Diversidad Alfa (Shannon-Wiener, Simpson y Pielou) del fitoplancton en la Plataforma Albacora del Lote Z1, en el año 2014.

De esta investigación podemos concluir que la variación espacio-temporal del fitoplancton marino en la plataforma Albacora, registró mayor riqueza y abundancia en las estaciones E-4 y E-5 durante el segundo trimestre. La distribución superficial trimestral del fitoplancton registra mayor riqueza y abundancia cuando la temperatura superficial alcanza un promedio de $25,5^{\circ} \mathrm{C}$, la salinidad 33,2 UPS, el pH 8,1 y el oxígeno disuelto 5,9 mg. $\mathrm{L}^{-1}$; valores que se encuentran dentro de los estándares de calidad de agua ECA y permiten el desarrollo de las especies en el área evaluada. Los índices de diversidad del fitoplancton reconocen el dominio de las diatomeas sobre los dinoflagelados en las estaciones E-1, E-2 y E-3 durante todos los trimestres del ańo y se reportan como bioindicadores del fitoplancton a Chaetoceros coarctatus Lauder, 1864, Guinardia striata (Stolterfoth) Hasle in Hasle \& Syvertsen, 1996 y Ceratium breve (Ostenfeld \& Schmidt) Schröder, 1906 relacionados con la presencia de las Aguas Ecuatoriales Superficiales (AES) (Fig. 14). 


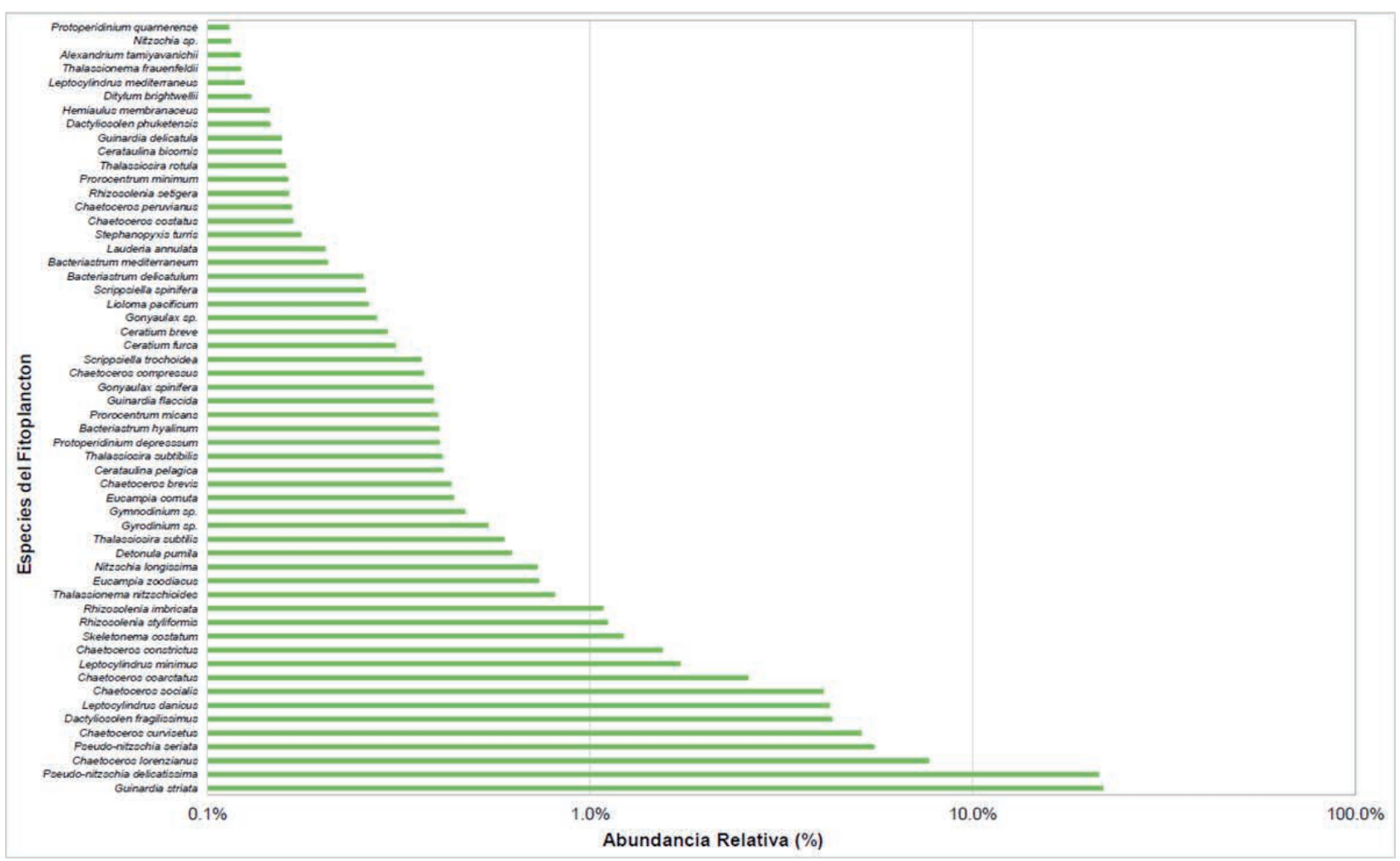

Figura 13. Abundancia Relativa (\%) del Fitoplancton registrado en los meses enero - diciembre 2014.

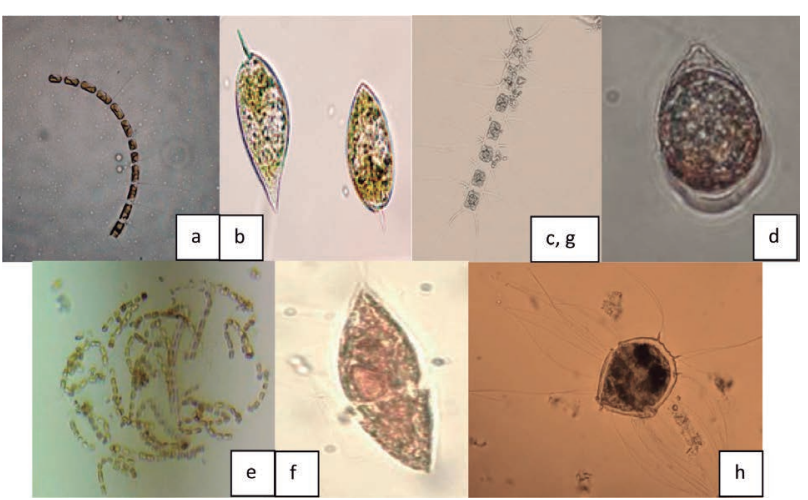

Figura 14. Especies de mayor abundancia por trimestre. Primer trimestre: (a) Chaetocerus curvisetus. (b) Prorocentrum micans. Segundo trimestre: (c) Chaetocerus lorenzianus. (d) Scrippsiella trochoidea. Tercer trimestre: (e) Chaetoceros sociales. (f) Gyrodinium sp. Cuarto trimestre: (g) Chaetocerus lorenzianus. (h) Gonyaulax spinifera.

\section{AGRADECIMIENTOS}

A Héctor Guevara Díaz, Gerente General de la empresa GEOLAB S.R.L, por permitirme utilizar los recursos necesarios para la presente investigación.

\section{REFERENCIAS BIBLIOGRÁFICAS}

Alcolado, P. 2001. Diversidad y bioindicación ambiental en el mar. Revista del Instituto de Oceanografía Ministerio de Ciencia Tecnología y Medio Ambiente (CITMA). Cuba. 10 p.

Armijos, S. 2007. Validez del plancton marino como indicador de condiciones oceanográficas en aguas ecuatorianas. Facultad de Ciencias Naturales. Universidad de Guayaquil. 173 pp.

Balech, E. \& Ferrando, H. 1964. Fitoplancton marino. Ed. Universitaria de Buenos Aires. Cap XII. pp. 114-122.

Basilio, V. 2014. Distribución y abundancia del Fitoplancton (Diatomeas y Dinoflagelados) en la Bahia de Santa Elena (Salinas-Ecuador) de juliodiciembre del 2013 (Licenciatura). Universidad Estatal Península de Santa Elena, Facultad Ciencias del Mar. 241 pp.

Coello, D.; Prado, M.; Cajas, J. \& Cajas, L. de. 2010. Variabilidad del plancton en estaciones fijas frente a la costa ecuatoriana. Revista de Ciencias del Mar y Limnología, 4: 23-43. 
Delgado, E. 2018. Características del fitoplancton del mar peruano verano 2014 crucero 1402-04. Boletín Instituto del mar del Perú, 33: 65-72.

Delgado, E.; Chang, F. \& Bernales A. 2015. Fitoplancton del mar peruano en el verano 2008. Informe Instituto del Mar de Perú, 42:172-177.

Hernández, A. \& Alcolado, P. 2014. Métodos para el estudio de la biodiversidad en ecosistemas marinos tropicales de Iberoamérica para la adaptación al cambio climático. E. Book. Instituto de Oceanología, La Habana-Cuba. 272 p.

INOCAR (Instituto Oceanográfico de la Armada). 2017. Derrotero de la costa Continental Insular del Ecuador. Instituto Oceanográfico de la Armada Ecuatoriana, $4^{\text {ta }}$ Ed., Guayaquil, Ecuador. Cap. I: 15 pp.

INVEMAR (Instituto de Investigaciones Marinas y Costeras). 2003. Hoja metodológica: indicador de la calidad ambiental de las aguas marinas y costeras. Proyecto especifico: Formulación y diseño del Sistema de Gestión de Indicadores Ambientales Marinos y Costeros de Colombia (Sigen). Primera fase. Instituto de Investigaciones Marinas y Costeras (INVEMAR), Santa Marta. 64 p

Lanza-Espino, G.; Hernández Pulido, S. \& Carbajal Pérez, J. 2011. Organismos indicadores de la calidad del agua y de la contaminación (bioindicadores) ( $2^{\mathrm{da}} \mathrm{ed}$.). México, D.F. Plaza \& Valdés, S.A. de C.V. 633 pp.

Magurran, A.E. 1988. Ecological diversity and its measurement. Book $1^{\text {st }} \mathrm{Ed}$. published by Croom Helm Ltd. USA. 175 p.

MINAM (Ministerio del Ambiente). 2008. Estándares Nacionales de Calidad Ambiental para Agua. Decreto Supremo No 002-2008-MINAM. Ministerio del Ambiente, Lima - Perú.

Mohan, R.; Shanvas, S.; Thamban, M. \& Sudhakar, M. 2006. Spatial distribution of diatoms in surface sediments from the Indian sector of Southern Ocean. Current Science, 91: 1495-1502.

Montero, P. 2013. Calidad ambiental de los ecosistemas acuáticos de la Región Tumbes, 2007. Boletín del Instituto del Mar Perú, 40: 240-253.

Morón, O. 2000. Características del ambiente marino frente a la costa peruana. Boletín del Instituto del Mar Perú, 19: 179-204.
Ochoa, N. \& Gómez, C.O.1997. Dinoflagelados del mar peruano como indicadores de masas de agua durante los años 1982 a 1985. Boletín IMARPE, 6: 1-60.

Ordinola, Q. 2017. Fitoplancton superficial entre las coordenadas $03^{\circ} 23^{\prime} y$ los $06^{\circ} 00^{\prime}$ Latitud Sur (Licenciatura). Universidad nacional de Piura, Facultad de Ciencias. 103 pp.

Pesantes, F. 1983. Los Dinoflagelados como Indicadores de "El Niño" en el mar Ecuatoriano. Acta Oceanográfica del Pacífico, 2: 85-117.

Prado-España, M.; Trócoli-Ghinaglia, L. \& Cajas-Flores, J. 2017 Análisis del estado trófico de la zona costera de la provincia de Guayas-Ecuador. Boletín de investigaciones marinas y costeras, 46: 91-114.

Rice, E. W. \& American Public Health Association (APHA). 2012. Standard methods for the examination of water and wastewater. $22^{\text {th }} \mathrm{Ed}$. Washington, DC: American Public Health Association.

Round, F. E.; Crawford, R. M. \& Mann, D.G. 2000. The diatoms: biology \& morphology of the genera. Cambridge: Cambridge University Press.

Ruppert, E.; Barnes, R. \& Salido, J. 1996. Zoología de los invertebrados (6 ${ }^{\text {th }}$ ed.). pp. 2-5. México: McGraw-Hill.

Ruiz, L. 2017. Fitoplancton superficial en La Caleta Los Órganos (Licenciatura). Universidad nacional de Piura, Facultad de Ciencias. 105 p.

Tarazona, J.; Gutierrez, D.; Paredes, C. \& Indacochea, A. 2003. Una revisión y desafíos para la investigación en biodiversidad marina en Perú. Gayana, 67: 206-231.

Tapia, M.E. 2012. Variabilidad estacional del fitoplancton y su relación con los parámetros ambientales en el estuario interior de Esmeraldas durante los años 2004-2005. Acta Oceanográfica del Pacífico, 17: 41-65.

Torres, G. \& Tapia, M. 2000. Distribución del Fitoplancton en la región costera del Mar Ecuatoriano, durante diciembre 2000. Acta Oceanográfica del Pacifico, 11: 31-41.

Utermöhl, H. 1958. Zur vervollkomnung der quantitativen phytoplankton methodik. Mitt Verhandlungen des Internationalen Verein Limnologie, 9:1-38. 
Vera, E. 2014. Incidencia de nutrientes y la TSM en proliferación de Dinoflagelados en dos sectores marino - costeros de la Provincia de Santa Elena (Maestría). Universidad de Guayaquil, Facultad de Ciencias Naturales. 144 pp.

Villanueva, P. 1997. Caracterización del Área Costera de Tumbes por organismos del fitoplancton durante 1990-1991. Tesis para optar el título de Biólogo Universidad Nacional de Piura. 87pp.
Zuta, S. \& Guillén, O. 1970. Oceanografía de las aguas costeras del Perú. Instituto del Mar del Perú Boletín IMARPE, 2: 157-324.

Received May 20, 2019.

Accepted June 22, 2019. 